\title{
Viscoelastic Relaxation and Recovery of Tendon
}

\author{
Sarah E. Duenwald, ${ }^{1}$ Ray Vanderby Jr., ${ }^{1,2}$ and Roderic S. Lakes ${ }^{1,3}$ \\ ${ }^{1}$ Department of Biomedical Engineering, University of Wisconsin-Madison, Madison, WI 53706-1687, USA; ${ }^{2}$ Department of \\ Orthopedics and Rehabilitation, University of Wisconsin-Madison, Madison, WI 53706-1687, USA; and ${ }^{3}$ Department of \\ Engineering Physics, University of Wisconsin-Madison, 1500 Engineering Drive, 541, Madison, WI 53706-1687, USA
}

(Received 7 July 2008; accepted 27 March 2009; published online 8 April 2009)

\begin{abstract}
Tendons exhibit complex viscoelastic behaviors during relaxation and recovery. Recovery is critical to predicting behavior in subsequent loading, yet is not well studied. Our goal is to explore time-dependent recovery of these tendons after loading. As a prerequisite, their straindependent viscoelastic behaviors during relaxation were also characterized. The porcine digital flexor tendon was used as a model of tendon behavior. Strain-dependent relaxation was observed in tests at $1,2,3,4,5$, and $6 \%$ strain. Recovery behavior of the tendon was examined by performing relaxation tests at $6 \%$, then dropping to a low but nonzero strain level. Results show that the rate of relaxation in tendon is indeed a function of strain. Unlike previously reported tests on the medial collateral ligament (MCL), the relaxation rate of tendons increased with increased levels of strain. This strain-dependent relaxation contrasts with quasilinear viscoelasticity (QLV), which predicts equal time dependence across various strains. Also, the tendons did not recover to predicted levels by nonlinear superposition models or QLV, though they did recover partially. This recovery behavior and behavior during subsequent loadings will then become problematic for both quasilinear and nonlinear models to correctly predict.
\end{abstract}

Keywords-Quasilinear viscoelasticity (QLV), Nonlinear superposition, Tendon.

\section{INTRODUCTION}

Like many tissues in the body, tendons exhibit viscoelastic, or time-dependent, behavior. When the tissue is held at a constant strain level, the stress in the tissue decreases, a phenomenon called stress relaxation. Conversely, when held at a constant stress level, strain in the tissue increases, known as creep. This viscoelasticity is thought to be a function of the main mechanical component, collagen, as well as other

Address correspondence to Roderic S. Lakes, Department of Engineering Physics, University of Wisconsin-Madison, 1500 Engineering Drive, 541, Madison, WI 53706-1687, USA. Electronic mail: duenwald@wisc.edu, lakes@engr.wisc.edu constituents of the extracellular matrix such as proteoglycans, glycoproteins, and water. Tendon collagen consists mainly of type I collagen fibers which are predominantly oriented parallel to the direction of load, or the long axis of the tendon. ${ }^{9,28}$

It is important to understand the viscoelasticity of tendon to accurately understand the behavior of a tendon. Creep and stress relaxation must be well defined in order to accurately predict how a tendon will behave under transient loadings, but a robust model of the tendon also requires an understanding of recovery behavior following removal of the load. Post-load recovery is not only important for understanding the unloaded tendon; it is also critical to tendon behavior under subsequent loadings. Failure to accurately account for the time-dependent recovery during unloaded periods will be a source of error when predicting behavior in response to future loads, especially if the unloaded period is short, and achieving reproducible data during serial testing. Although stress relaxation and creep behaviors of many tissues have been studied, including rat $\mathrm{MCL},{ }^{29}$ sheep digital tendon, ${ }^{33}$ bovine cornea, ${ }^{3}$ and the stapedial tendon in the middle ear, ${ }^{4}$ relatively few studies investigate the recovery periods following creep or stress relaxation testing. This omission leads to incomplete data to understand and model post-loading behavior in tendon.

The main research concerning the recovery period following loading is regarding the recovery of the spine and its discs following creep experiments. McGill and Brown performed creep testing on human subjects for $20 \mathrm{~min}$ and monitored the recovery after the removal of the load for $20 \mathrm{~min}^{21}$ Likewise, Eckstrom et al. induced creep in the porcine spine over $1 \mathrm{~h}$, and observed its recovery for an hour following the removal of load. ${ }^{8}$ Gedalia et al. performed similar testing in lumbar structures, loading them for $50 \mathrm{~min}$ and monitoring recovery after $50 \mathrm{~min} .{ }^{10}$ Finally, Solomonow et al. subjected cat spines to cyclic loading for 
$50 \mathrm{~min}$, and observed the recovery for $7 \mathrm{~h}^{32}$ Interestingly, each of these studies found that the recovery of creep in the spine requires more time than the duration over which the load was applied. However, with the different structure and function of the tendon, it is crucial to perform experiments on tendon itself rather than draw conclusions from spine behavior.

Surgical applications have also sparked research regarding tendon viscoelasticity. Graf et al. found that recovery from 10 -min stress relaxation testing was not complete after 30 min. ${ }^{11}$ Understanding creep and recovery of a tendon graft is important to ensure proper joint kinematics and maximize success in joint reconstructions.

Knowledge of the biomechanical behavior of the tendons and ligaments is also important for understanding, quantifying, and treating injuries in these tissues. Their behaviors are important when considering the wide use of grafts, whether in an autograft, synthetic graft, or allograft, as any such graft should ideally match the properties of the predamaged tendon or ligament for optimal function. Increased laxity could allow abnormal and extreme movement of the joint, leading to injury of surrounding tissue. Insufficient laxity could limit movement and result in tearing of the graft under normal movement conditions. A distinction between tendon and ligament behavior is also important when considering grafts. Though often viewed as surgically interchangeable, tendons and ligaments serve different biomechanical functions and have unique behaviors. Even subtle differences may be important for grafting and tissue engineering applications. The goal of this study therefore is to use the porcine digital flexor tendon as a model to characterize stress relaxation over a range of strains, comparing its behavior to that found in other tendon and ligament studies, and examine the timedependent recovery behavior of the tendon following the removal of a step load.

\section{MATERIALS AND METHODS}

Forty-three digital flexor tendons were carefully dissected from porcine legs obtained from a local abattoir. Ten were allocated to each experiment (as described in the following paragraphs), and three were used for a repeatability study (described later). The tendon was removed from the muscle belly, but remained attached to the bone at the distal insertion site. All excess tissue, including muscle tissue and the tendon sheath, was carefully removed from the tendon. The bone was potted in lightweight filler hardened with a cream hardener for gripping in the test machine. The tendons were kept hydrated with PBS saline solution throughout harvesting and processing. Tendons were then wrapped in saline-soaked gauze, covered with aluminum foil, and sealed in plastic bags to be stored in a $-30{ }^{\circ} \mathrm{C}$ freezer until time of testing. Investigators report that such careful freezing procedures have little effect on the biomechanical properties of collagenous tissues such as tendon and ligament. ${ }^{14,22}$

All experiments were performed using a servohydraulic mechanical test system (Bionix 858; MTS, Minneapolis, MN) in conjunction with a computer to collect data. The load frame was equipped with a lower block designed to hold the potted bone and an upper soft tissue grip designed to hold the tendon. The upper grip utilized a holder for dry ice to provide a secure grip on the tendon fibers, and is denoted a "cryogrip." This cryogrip was connected to a $50 \mathrm{lb}$ load cell (Eaton Corporation) with data collected on a PC. The lower block remained stationary during test runs, while the cryogrip moved to the specified displacement. All strain was measured as the grip-to-grip elongation divided by the undeformed length of the specimen. The lower block and tendon were contained in a bath so that throughout testing the tendon was submerged in physiologic buffer solution at ambient room temperature.

All testing began with a preload of $1 \mathrm{~N}$ to remove slack in the tendon and a preconditioning protocol in which the tendon was stretched to $2 \%$ strain in a sinusoidal wave for $20 \mathrm{~s}$ with a period of $2 \mathrm{~s}$. After preconditioning, the specimen was allowed to recover for $5 \mathrm{~min}$ before being again stretched to $2 \%$ strain and held for $100 \mathrm{~s}$ (see Fig. 1), after which it was un-

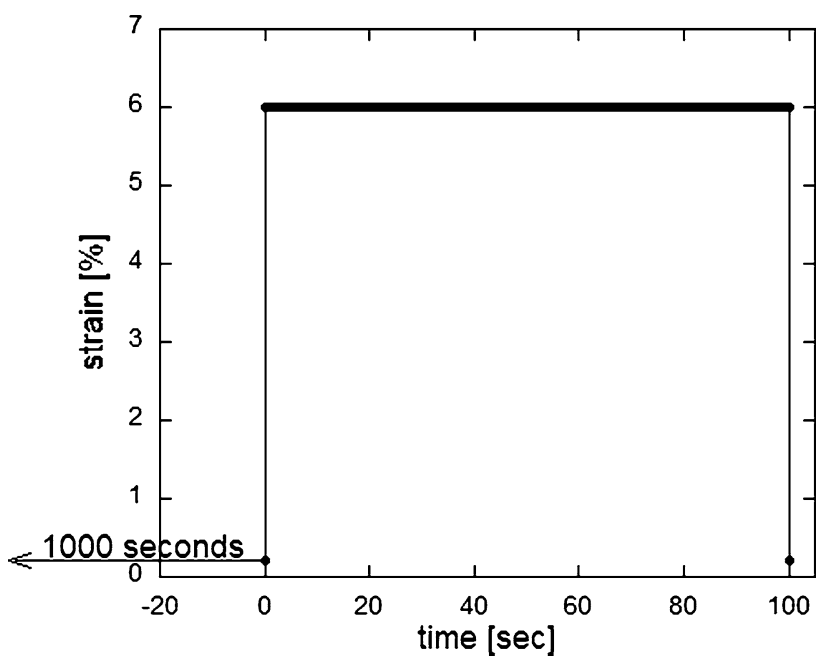

FIGURE 1. Input waveform for single relaxation analysis without observed recovery. This waveform was performed at $1,2,3,4,5$, and $6 \%$ strains (only $6 \%$ strain input shown here) for the relaxation at various strains experiments and at $2 \%$ strain during preconditioning. 
loaded for $1000 \mathrm{~s}$, or 10 times the length of relaxation, prior to further testing. Strain was treated as a step rather than a ramp, with the zero time point assigned halfway through the rise time, and data collection beginning at $t=2.5 t_{\mathrm{r}}$, or about $0.1 \mathrm{~s}$, where $t_{\mathrm{r}}$ is the rise time of the machine. This experimental design follows Lakes' example for creep. ${ }^{15}$

The cross-sectional area of the tendon was assumed to be elliptical, and was calculated (prior to testing) from micrometer measurements of the long and short axes when the tendon was under preload.

Stress in the tissue was computed as:

$$
\sigma(t)=F(t) / A_{0},
$$

where $F(t)$ is the force as a function of time, and $A_{0}$ is the cross-sectional area of the tendon. The relaxation function was calculated via:

$$
E(t)=\sigma(t) / \varepsilon,
$$

where $\sigma(t)$ is the stress defined in Eq. (1), and $\varepsilon$ is the constant strain level to which the tendon is pulled.

The basic form of the nonlinear superposition is:

$$
\sigma(\varepsilon, t)=\int E[t-\tau, \varepsilon(\tau)] \frac{d \varepsilon(\tau)}{d \tau} d \tau,
$$

where $E(t, \varepsilon)$ is the relaxation function (defined in Eq. 2). With the use of the discrete step strain function, the nonlinear superposition prediction becomes:

$$
\sigma(t)=\varepsilon_{1} E\left(t, \varepsilon_{1}\right)-\left(\varepsilon_{1}-\varepsilon_{2}\right) E\left(t-t_{1}, \varepsilon_{2}\right),
$$

where $t$ is the time from the start of stress relaxation at strain level $\varepsilon_{1}$ and $t_{1}$ is the time at which recovery at strain level $\varepsilon_{2}$ begins.

The basic equation for stress in QLV is:

$$
\sigma(t)=\int_{0}^{t} E_{t}(t-\tau) g(\varepsilon) \frac{d \sigma}{d \varepsilon} \frac{d \varepsilon(\tau)}{d \tau} d \tau .
$$

where $E(t, \varepsilon)$ has been separated into the product of a time-dependent modulus, $E_{t}(t)$, and $g(\varepsilon)$, which represents the nonlinear strain dependence (independent of time).

Since we are using a discrete step strain function, we utilize the form:

$$
\sigma(t)=\left(\varepsilon_{1}\right) E_{t}(t) g\left(\varepsilon_{1}\right)-\left(\varepsilon_{1}-\varepsilon_{2}\right) E_{t}\left(t-t_{1}\right) g\left(\varepsilon_{2}\right)
$$

to describe the stress behavior predicted by QLV.

\section{Relaxation at Various Strains}

Ten porcine flexor tendons were tested in this portion of the experiment to examine the stress relaxation behavior that occurs as the tendon is tested over a range of strains. The tendons were tested at 1, 2, 3, 4, 5, and $6 \%$ strain in randomized order to ensure that the trends were due to differences in strain, not the testing order. Each relaxation test involved the tendon being stretched to the prescribed strain $(1,2,3,4,5$, or $6 \%)$ and held for $100 \mathrm{~s}$ prior to being released to the preload level to rest for $1000 \mathrm{~s}$ before the next test was run (see Fig. 1 for representative waveform). The tendon was then stretched to the next prescribed strain and released, in the same manner as just described, until all six strain levels $(1-6 \%)$ had been tested. Force data were recorded throughout the entire relaxation and recovery period on the PC simultaneously with time and strain recording. At the conclusion of the sixth test, data were exported for analysis.

\section{Relaxation and Recovery Comparison}

Ten porcine flexor tendons were tested to examine the recovery behavior during the period immediately following stress relaxation. Tendons were stretched to $2 \%$ strain, at which they were held for $100 \mathrm{~s}$, and then dropped to preload at which they rested for $1000 \mathrm{~s}$. They were then stretched to $6 \%$ strain for $100 \mathrm{~s}$ of relaxation, after which the strain was lowered to $2 \%$ for a $100 \mathrm{~s}$ period in which recovery could be observed (see Fig. 2 for waveform). The recovery was recorded at a low strain rather than zero strain, as zero strain would cause the tendon to become slack, preventing observation of recovery behavior with the load cell. The maximum strain of $6 \%$ was chosen as it would be high enough to allow adequate recovery at a nonzero

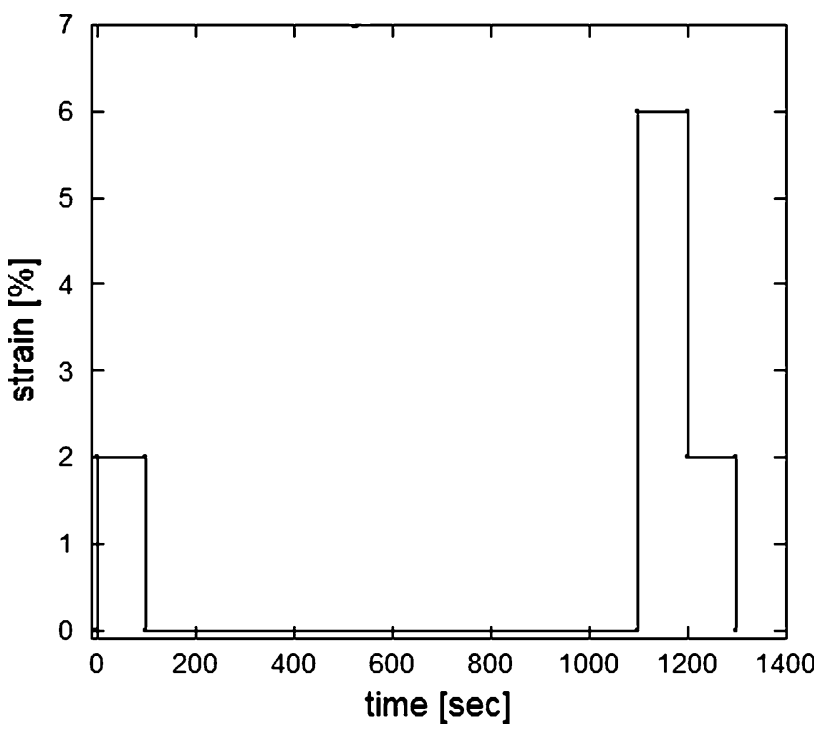

FIGURE 2. Input waveform for relaxation and recovery analysis. During such analysis, relaxation always took place at $6 \%$ strain, but the recovery portion was performed at 1,2 , and $3 \%$ strain. 
strain while still being low enough to avoid damage to or failure of the tissue.

After the recovery period, the specimen was released to its preload and allowed to rest for $1000 \mathrm{~s}$ before repeating the waveform. This was done until a total of three waveforms were completed on the tissue (to ensure a good data set from each specimen), and repeated for each of the 10 specimens. Force data were recorded throughout the entire relaxation and recovery period on the PC simultaneously with time and strain recording. $E(t)$ was calculated from the stress relaxation data at $6 \%$ and $2 \%$ strain, and curve fits of the $E(t)$ were calculated in order to make model predictions of the relaxation and recovery. Nonlinear superposition and QLV equations were then plotted with and compared to experimental data.

This procedure was repeated for 10 tendons for recovery at 3\% strain and 10 tendons for recovery at $1 \%$ strain (with the relaxation performed at $6 \%$ strain as before) for a total of 30 tendons (including the previous 10 in this section), where all previously mentioned components of $2 \%$ strain were replaced with $3 \%$ and $1 \%$ strain. This was done to determine whether the nonzero strain $(1,2$, or $3 \%)$ level determined the rate of recovery.

\section{Repeatability and Statistics}

In order to evaluate the repeatability of experiments on a particular specimen, three specimens underwent the relaxation (at $6 \%$ strain) and recovery (at $2 \%$ strain) procedures outlined in the previous section a total of five times. Load data were recorded over $100 \mathrm{~s}$ of relaxation and over $100 \mathrm{~s}$ of recovery. Load values at $0.1,1,10$, and $100 \mathrm{~s}$ were averaged between the relaxation runs for each tendon, and the standard deviation at each time point was measured; the process was then repeated for each of the recovery runs. This process allowed a quantification of repeatability not achievable through graphical representation alone; by analyzing the time points in each run it is possible to compare the similarity of each curve (small standard deviations ensure that the curves follow the same general path).

The deviation between different specimens was also of interest. Variations between stress relaxation curves and recovery curves from data collected from all 43 specimens (not just from the three involved in this study) were measured to get the full range of specimento-specimen variation. The average and standard deviation between the curves of these specimens were measured and recorded in a table. Student $t$-tests were conducted on $A$ and $n$ values to determine statistical significant differences (where a value of $p \leq 0.05$ is considered statistically significant).

\section{RESULTS}

Data from each of the various tests were graphed so that behavioral trends could be examined. Information from each tendon tested was collected and graphed, then categorized into subgroups based on the test(s) performed on the tissue.

\section{Relaxation at Various Strains}

Two types of graphs were constructed from the data collected for each tendon tested at various strains. First, a graph showing load vs. time (Fig. 3) was produced to describe the relative behavior at various strains. Next, the data were used to create a set of
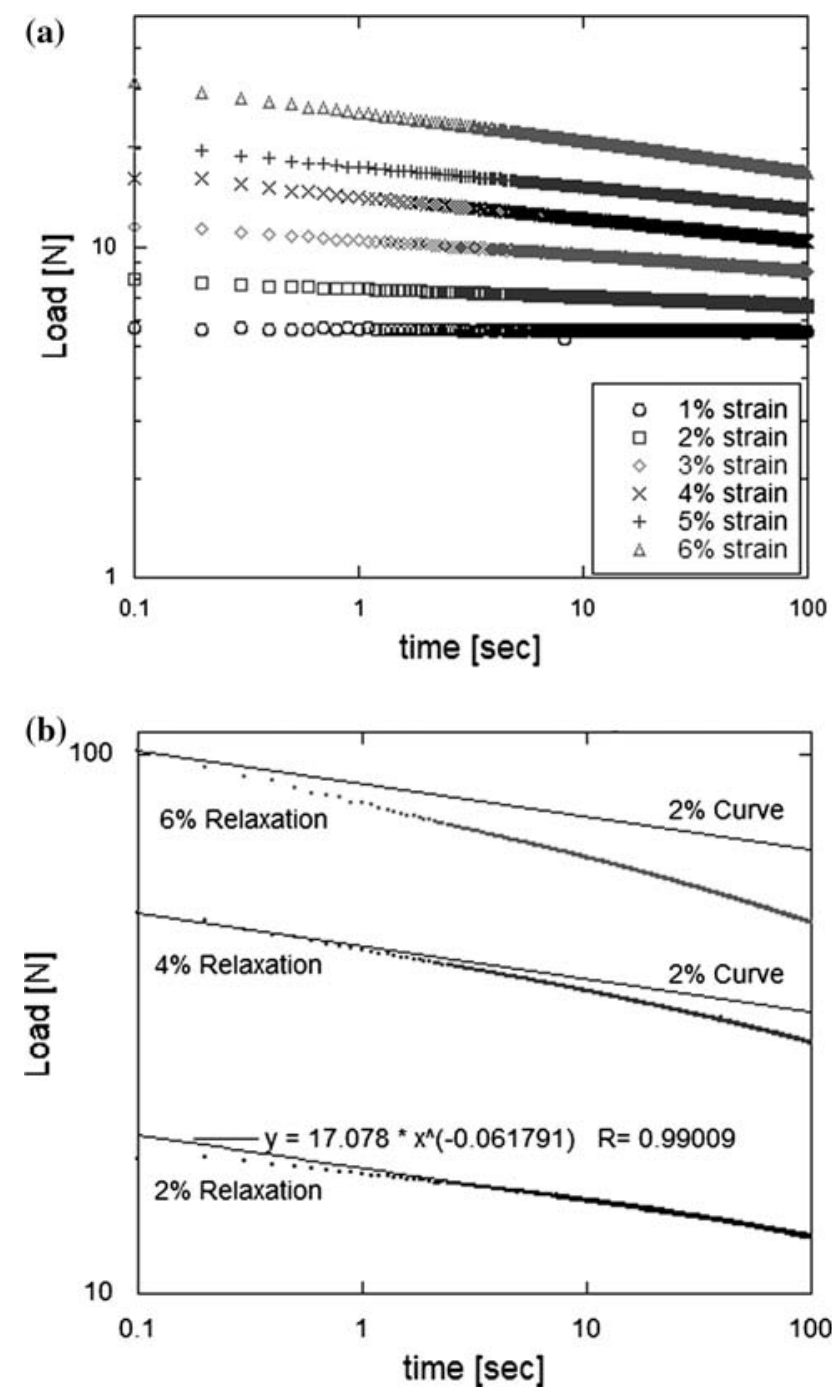

FIGURE 3. Load vs. time during relaxation of the tendon tissue at various strains, displaying the increasing time dependency with increasing strain. (a) All six strain levels, showing the converging nature of the curves. (b) 2,4 , and $6 \%$ strain curves, each data set fitted with the curve fit of the $2 \%$ strain. 


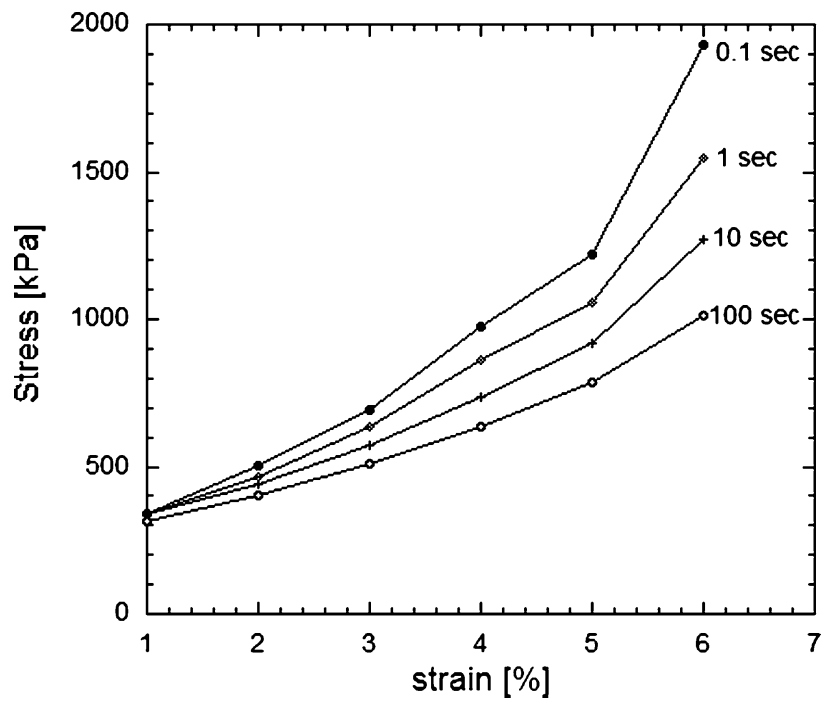

FIGURE 4. Isochronal curves showing the stress at each strain level for designated time point, displaying the strainstiffening behavior of the tendon.

isochronal stress vs. strain curves to display the strainstiffening behavior of the tendon (Fig. 4).

Figure $3 \mathrm{a}$ shows that the rate of relaxation (demonstrated by the slope of the log-log plot) increases with increasing strain. Thus, the tendon displays more viscoelastic behavior at the higher strains and more elastic behavior at lower strains. This trend is further displayed in Fig. $3 \mathrm{~b}$ as the curve fit of the $2 \%$ strain is plotted with the $4 \%$ and $6 \%$ strain data. This figure also displays that QLV is inadequate to model the relaxation behavior; QLV predicts that the rate of relaxation is independent of strain, ${ }^{27}$ which differs from our observed relaxation behavior.

The isochronal curves in Fig. 4 show that despite the higher rates of relaxation at higher strains, the tendon displays strain-stiffening behavior at all times (with an increase in strain on the tendon, the tendon becomes increasingly stiff), manifested by the concaveup slope of the curve. ${ }^{16}$

\section{Relaxation and Recovery Analysis}

Force vs. time information from the $6 \%$ strain and the $2 \%$ strain tests, as well as the force vs. time data gathered during the recovery period (when strain is reduced from $6 \%$ to $2 \%$ ), normalized for strain level (i.e., divided by their relative strain level), were plotted on a graph, and fitted with a power law equation (this was also performed for experiments using $6 \%$ and $3 \%$ or $6 \%$ and $1 \%$ strain). When plotted on a log-log scale (see Fig. 5), the results follow straight line trends, indicating that both relaxation and recovery curves can be approximated by the power law equation:

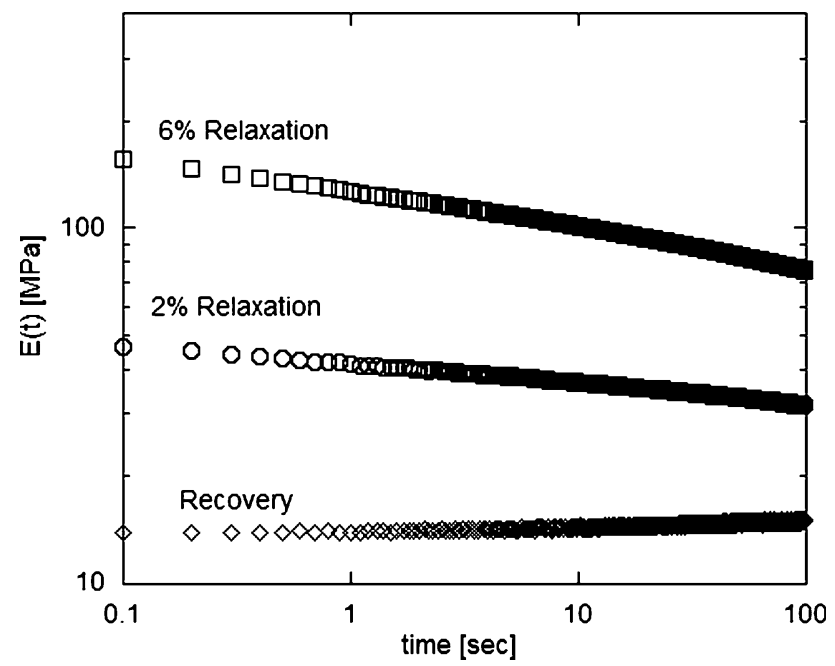

FIGURE 5. Relaxation and recovery results from one complete test of the tendon, showing the increased slope of the relaxation curve compared to the recovery curve.

$$
\frac{\sigma}{\varepsilon_{0}}=A t^{n}
$$

where $A$ has units of $\mathrm{Pa}$ and $t$ has units of seconds. The slopes of the straight lines of the relaxation data on these plots indicate the power, or $n$ value, of the data, and thus the magnitude of $n$ indicates how rapidly relaxation or recovery occurs in time. In agreement with the previous results of relaxation at various strains, the absolute value of the power of the $6 \%$ strain relaxation curve (in this example, $n=0.115$ ) was higher than that of the $2 \%$ strain relaxation curve (in this example, $n=0.060$ ). Interestingly, the absolute value of the power of the recovery curve (in this example, $n=0.017$ ) was much smaller than either of the relaxation curves. This indicates that the recovery occurs at a much slower rate than relaxation, even the relaxation at the lower strains. Relative rates at different strain levels will be discussed further in the next section.

Figure 6 contains both the nonlinear superposition prediction and the QLV prediction based on relaxation curves performed at $6 \%$ and $3 \%$ strain. Neither nonlinear superposition nor QLV accurately predict the recovery behavior. Nonlinear superposition over-predicted the recovery (at the highest stress) by $218.6 \pm 58.6 \%$, and QLV over-predicted the recovery (at the highest stress) by $266.9 \pm 50.9 \%$.

While recovery during the $100 \mathrm{~s}$ period immediately following stress relaxation was slow and did not come to completion during the 100 -s period, it was observed that, following the $1000 \mathrm{~s}$ of unloading between successive tests, the tendon recovered to within $0.5 \mathrm{~N}$ of the original preload in all cases. 

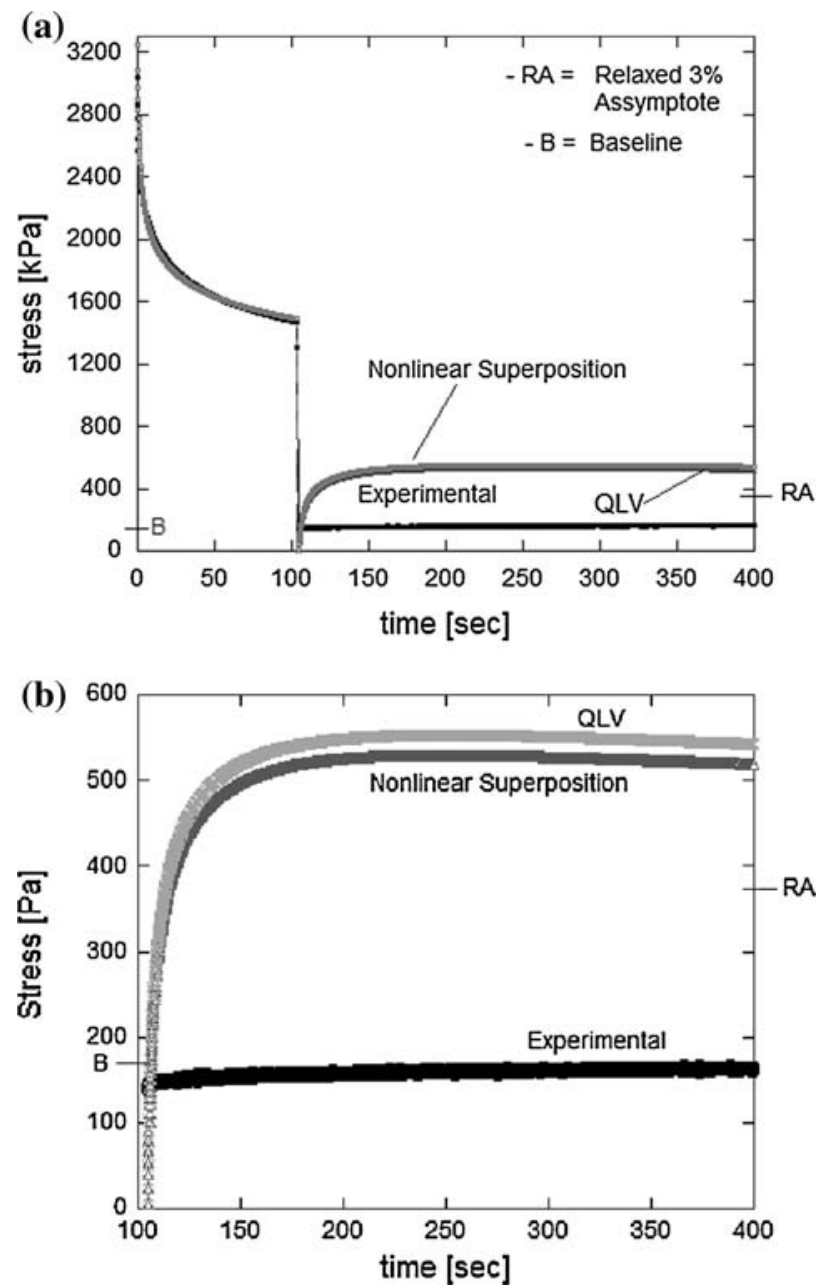

FIGURE 6. Experimental data compared to nonlinear superposition and QLV models. The baseline is denoted by " $B$ " and the relaxed $3 \%$ asymptote (lowest level reached during relaxation) is denoted by "RA." (a) Relaxation and recovery curves based on experimental data, nonlinear superposition model, and QLV model. (b) Recovery portion of the data, demonstrating that neither QLV nor nonlinear superposition are accurate predictions of recovery behavior, and QLV is the worse fit of the two.

\section{Repeatability and Statistics}

It was observed that the relaxation (Fig. 7a) and recovery (Fig. $7 b$ ) tests run on a single specimen were quite repeatable, with small deviations between either recovery curves or relaxation curves (Tables 1 and 2). There was, however, a larger amount of variability between different tendons, leading to larger standard deviations in both the $A$ and $n$ values (Table 3). Even with the larger standard deviations, many of the $A$ and $n$ values were significantly different between strain levels. $A$ values, which represent the initial stress magnitude for a given strain level, were significantly higher for stress relaxation curves at $6 \%$ strain than for relaxation at $3 \%$ strain $(p=0.0418)$ and at $2 \%$ strain
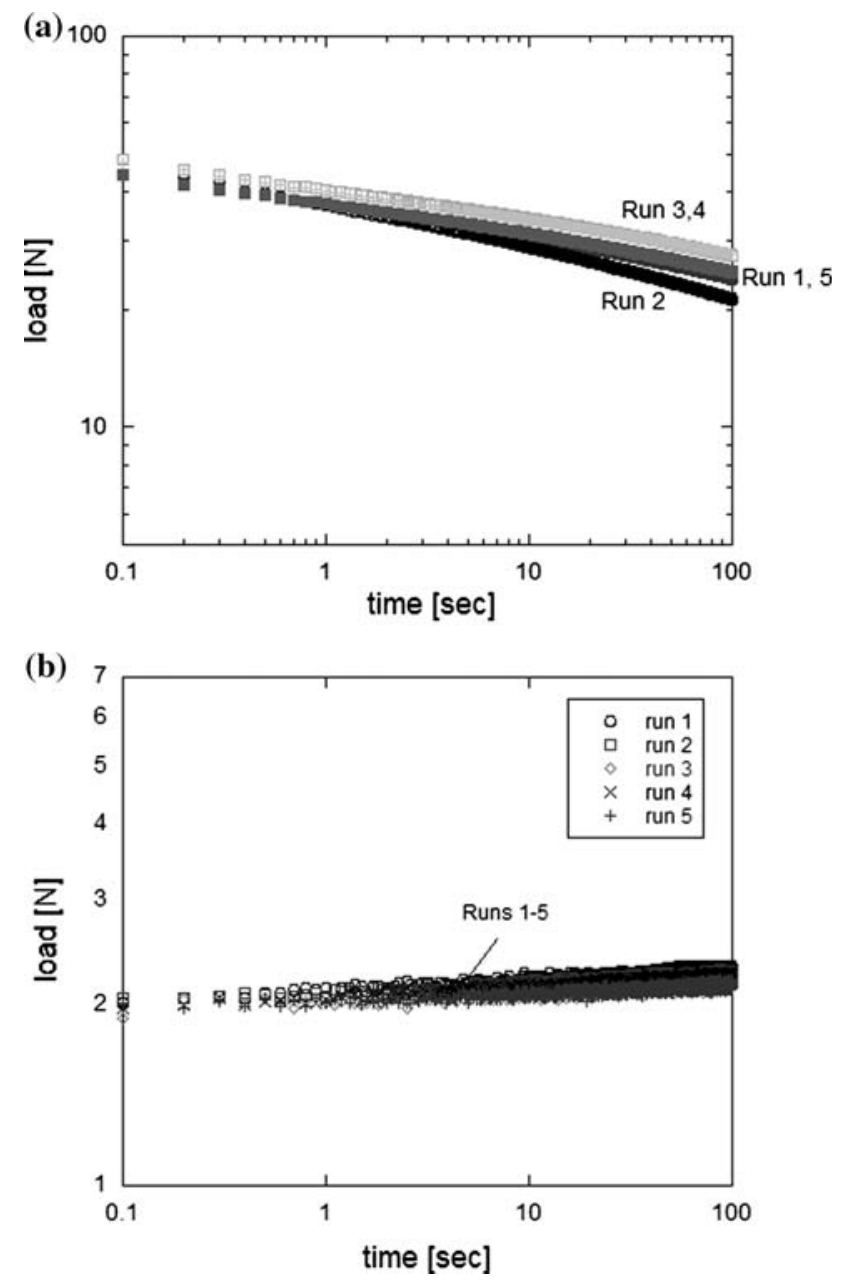

FIGURE 7. Relaxation (a) and recovery (b) curves on a single specimen. The relaxation and especially the recovery are very repeatable between test runs on a single specimen.

TABLE 1. Repeatability statistics on five relaxation tests for a single specimen.

\begin{tabular}{lcccc}
\hline & \multicolumn{4}{c}{ Relaxation } \\
\cline { 2 - 5 } & \multicolumn{4}{c}{ Loads (N) at time points } \\
\cline { 2 - 5 } & $0.1 \mathrm{~s}$ & $1 \mathrm{~s}$ & $10 \mathrm{~s}$ & $100 \mathrm{~s}$ \\
\hline Averages (N) & 84.64 & 72.02 & 61.22 & 46.62 \\
Standard deviation (N) & 7.483 & 4.548 & 4.003 & 3.028 \\
\% of average & 8.840 & 6.315 & 6.539 & 6.495 \\
\hline
\end{tabular}

$(p=0.0003)$, and were also significantly higher for relaxation at $3 \%$ strain vs. $2 \%$ strain $(p=0.050)$. $A$ values were significantly lower for recovery curves at $1 \%$ strain vs. both $3 \% \quad(p=0.0048)$ and $2 \%$ $(p<0.0001)$ strains, but were not significantly higher for $3 \%$ strain compared to $2 \%(p=0.3121)$. The $n$ values, which represent the rate of relaxation or recovery at a given strain level, were significantly 
higher for relaxation at $6 \%$ strain than at 3\% strain $(p<0.0001)$ and $2 \%$ strain $(p<0.0001)$. The $n$ values were also significantly higher for relaxation at $3 \%$ strain than at $2 \%$ strain $(p=0.0291)$. The rates of recovery ( $n$ values) were not significantly higher at 3\% strain than at $2 \%$ strain $(p=0.1115)$, but were significantly higher at $3 \%$ than at $1 \%$ strain $(p=0.0059)$; they were also significantly higher at $2 \%$ strain than at $1 \%$ strain $(p=0.0008)$. The rate of relaxation at $6 \%$ strain was significantly higher than the rate of recovery at 1,2 , or $3 \%$ strain $(p<0.0001$ in all three cases).

\section{DISCUSSION}

Our experimental results show that both relaxation and recovery of the porcine digital flexor tendon over $100 \mathrm{~s}$ of loaded behavior can be well approximated by a power law in time, but the recovery is not predicted by a simple nonlinear superposition equation or by the more complex QLV theory. The experimental recovery proceeds at a much slower rate than relaxation, and much slower than predicted by nonlinear superposition or QLV. Also deviating from the theory of QLV was the presence of a strain-dependent relaxation rate, as demonstrated by Figs. 3a and 3b. The strain-stiffening tendency of the isochronal stress-strain curves (Fig. 4) suggest that the observed increased relaxation at higher strain levels was not due to slippage of the tendon in the grips. Such behavior would result in

TABLE 2. Repeatability statistics on five recovery tests for a single specimen.

\begin{tabular}{lllll}
\hline & \multicolumn{4}{c}{ Recovery } \\
\cline { 2 - 5 } & \multicolumn{4}{c}{ Load (N) at time points } \\
\cline { 2 - 5 } & $0.1 \mathrm{~s}$ & $1 \mathrm{~s}$ & $10 \mathrm{~s}$ & $100 \mathrm{~s}$ \\
\hline Average load $(\mathrm{N})$ & 1.988 & 2.051 & 2.108 & 2.206 \\
Standard deviation $(\mathrm{N})$ & 0.0576 & 0.0536 & 0.0640 & 0.0483 \\
\% of average & 2.900 & 2.615 & 3.035 & 2.188 \\
\hline
\end{tabular}

strain-softening (marked by a concave-down slope of the line) behavior at higher strains.

Our study is not the first to demonstrate the limitations of QLV in soft tissues. A major limitation noted by others is the assumption of a linear material response, ${ }^{34}$ manifesting itself in a single reduced relaxation function over a range of strain levels ${ }^{26}$ or constant amplitude of viscous effects over a range of frequencies. ${ }^{17}$ The QLV model was inaccurate at high strain rates during experiments performed by Woo et al. ${ }^{35}$ and has also been shown by investigators to under-predict hysteresis ${ }^{25,36}$ and early stress relaxation. $^{7}$

Physiologic strain ranges up to $8 \%$ in vivo, ${ }^{20}$ so it was assumed that a grip-to-grip strain of $6 \%$ would be below the damage threshold, and thus would allow recovery of the tissue. It has been suggested by Magnusson et al. that tendon is less likely to deform than the muscle-tendon unit during loading. ${ }^{20}$ In fact, Lieber et al. noted that while passive stretch in the physiologic range can be up to $8 \%$ strain, only $2 \%$ of that strain occurs in the tendon. ${ }^{18}$ This indicates that, in vivo, tendon may experience lower strains than used in this study (up to 6\%), and perhaps recovers more slowly from such strains. The isochronal curves in Fig. 4 indicate that tendon is still in the pre-damage "toe" region of the load-strain curve, and the stresses induced at $6 \%$ strain were much lower than the ultimate stress of the tendon, which is $80-90 \mathrm{MPa}^{31}$ (Fig. 6 displays a maximum stress of less than $1 \mathrm{MPa}$ ). Possible explanation for the low rate of recovery include incomplete recovery of water lost during loading, a phenomenon discussed by Han et al., ${ }^{12}$ or failure of proteoglycan-collagen interactions, which may be capable of storing some energy elastically. ${ }^{23,24}$ The ability of tendon to nearly recover to preload in every instance indicates that there is little or no plasticity.

The relaxations at various strains demonstrate a higher rate of relaxation at higher strains, and a lower rate of relaxation at lower strains. This behavior is similar to results found for sheep digital tendons, ${ }^{33}$ porcine digital flexor tendon, ${ }^{5}$ mouse tail tendons, ${ }^{9}$ and

TABLE 3. $\boldsymbol{A}$ and $\boldsymbol{n}$ values for relaxation and recovery curves for all specimens.

\begin{tabular}{lccccc}
\hline & \multicolumn{2}{c}{ Relaxation } & & \multicolumn{2}{c}{ Recovery } \\
\cline { 2 - 3 } \cline { 5 - 5 } Strain level (\%) & $A(\mathrm{MPa})$ & $n$ & & $A(\mathrm{MPa})$ & $n$ \\
\hline 6 & $83.3 \pm 50.5$ & $-0.113 \pm 0.026$ & - & - \\
3 & $59.7 \pm 47.5$ & $-0.078 \pm 0.031$ & & $13.2 \pm 11.3$ & $0.044 \pm 0.044$ \\
2 & $38.3 \pm 31.2$ & $-0.051 \pm 0.027$ & & $11.5 \pm 4.46$ & $0.027 \pm 0.019$ \\
1 & - & - & $2.70 \pm 1.79$ & $0.005 \pm 0.002$ \\
\hline
\end{tabular}

Respective number of specimens for each category: relaxation at $6 \%(n=43)$, relaxation at $3 \%(n=20)$, relaxation at $2 \%(n=20)$, recovery at $3 \%(n=10)$, recovery at $2 \%(n=13)$, and recovery at $1 \%(n=10)$. 
bovine cornea. ${ }^{3}$ This behavior is opposite, however, to results found for the MCL of rabbit, ${ }^{13}$ human, ${ }^{2}$ and rat. ${ }^{29}$ While some differences may be due to gripping differences (tendons have a soft-tissue grip, while ligament involve two bone grips), different insertion sites (ligament testing incorporates two insertion sites, tendon only one), or strain measurement differences (gripto-grip measurement vs. strain tracking using optical markers), such results hint at biomechanical differences between tendons and ligaments, which should be pursued further in the future. There are also large differences between tendons that serve different functions. Batson et al. discovered significant material and structural differences between the superficial digital flexor tendon and the common digital extensor tendon of the horse, including differences in water content, ultimate strain, and both glycosaminoglycan and chondroitin contents. ${ }^{1}$ Rumian et al. found differences in the extracellular matrix between an assortment of tendons and ligaments in sheep, finding differences not only between the tendon and ligament groups, but also between the various ligaments and tendons themselves, including fibril diameter distributions, water content, and glycosaminoglycan content. ${ }^{30}$ These findings show that tendon and ligament properties vary with position and function in the body. Whether these variations are due to site-specific mechanical adaptation or are predetermined genetically remains unknown.

The recovery behaviors following creep and/or relaxation testing in other biological tissues, particularly the spine, show a similarly slow rate. Not only did the spines fail to recover during an unloading period equal in length to the loading period, ${ }^{8,21}$ most still had not completely recovered in unloaded periods double or more the length of time of loading. Gedalia et al. observed that the lumbar structures failed to recover after more than double the amount of time allotted for creep testing. ${ }^{10}$ Likewise, Solomonow et al. observed only $92 \%$ recovery after a full $7 \mathrm{~h}$ of recovery following $50 \mathrm{~min}$ of cyclic loading in the cat spine. ${ }^{32}$ Following 1-h creep tests in wrist ligaments, Crisco et al. observed that after $2 \mathrm{~h}$ of unloading the ligaments had not yet fully recovered. However, after $24 \mathrm{~h}$ of unloading, the wrist ligaments had fully recovered from creep, suggesting that other biological tissues (including tendon) can fully recover if given adequate time. ${ }^{6}$

An interesting behavior was also discovered in microtubule networks by Lin et al. An experiment found that following creep testing, the extent of the recovery of the microtubule network was dependent on the nature of the network. They found that the crosslinked microtubule network recovered completely, where the physically entangled microtubule network had a residual strain of 0.12 (after creeping to a strain of approximately 0.55). ${ }^{19}$ This phenomenon is intriguing to investigators, as it suggests that the microstructure of the tendon may be a factor influencing its recovery, an idea that could be pursued further in future experiments.

Studies of nonbiological viscoelastic polymers can help give insight into the behavior of biological polymeric components such as collagen. A study by Zhang and Moore investigated the creep and relaxation behavior of high density polyethylene, as well as the recovery of the polymer following the removal of load. ${ }^{37}$ Like tendon, the polyethylene exhibited nonlinear behavior during both uniaxial loading and unloading. Also similar to the tendon, following removal of load, the polyethylene recovered continuously, but at a rate slower than that of relaxation or creep. Previous studies on basic polymers such as polyethylene loaded in the same way as tendon may then provide insight into behaviors of which can be related to biological polymers like collagen, the major mechanical component of tendon, and provide input into future models of tendon behavior.

The understanding of viscoelastic properties of tissues is crucial to understanding their biomechanical function in the body. In vivo, the time-dependent properties of tendon affect their ability to convert muscle contraction into skeletal movement as well as positional stability of the body. The recovery behavior of tendon elucidates this effect. Slow recovery of tendon after loading means it will likely not achieve full recovery prior to subsequent loading, and it will deform more (from its original length) under similar loads (in creep scenarios); failure to recover completely from a stress relaxation scenario results in a slightly longer tendon, reducing the efficiency of the muscletendon contraction.

This study examined the viscoelastic behavior of the porcine digital flexor tendon, investigating the strain dependency of stress relaxation and focusing on the largely unexamined recovery that follows unloading. We observed that the rate of stress relaxation is dependent on strain level, which does not follow the QLV model. We also found that the strain-dependence of stress relaxation in our tendon model, as well as those found in the literature (increasing rate with increasing strain), has a trend opposite that of ligament studies found in the literature (decreasing rate with increasing strain). By recording the recovery from load at a low but nonzero strain level, we were able to monitor an entire recovery curve over $100 \mathrm{~s}$. This allowed for analysis of the recovery behavior of tendon, which progresses at a rate slower than relaxation and is not well predicted with current viscoelastic models for tendon. This study expands our understanding of tendon behavior, provides data for 
more robust models of tendon behavior, and demonstrates the complex biomechanical challenges for replacement tissues.

\section{ACKNOWLEDGMENTS}

This work was funded by NSF award 0553016 . The authors thank Ron McCabe for his technical assistance.

\section{REFERENCES}

${ }^{1}$ Batson, E. L., R. J. Paramour, T. J. Smith, H. L. Birch, J. C. Patterson-Kane, and A. E. Goodship. Are the material properties and matrix composition of equine flexor and extensor tendons determined by their functions? Equine Vet. J. 35(3):314-318， 2003. doi:10.2746/042516403 776148327.

${ }^{2}$ Bonifasi-Lista, C., S. P. Lake, M. S. Small, and J. A. Weiss. Viscoelastic properties of the human medial collateral ligament under longitudinal, transverse, and shear loading. $J$. Orthop. Res. 23:67-76, 2005. doi:10.1016/j.orthres.2004. 06.002 .

${ }^{3}$ Boyce, B. L., R. E. Jones, T. D. Nguyen, and J. M. Grazier. Stress-controlled viscoelastic tensile response of bovine cornea. J. Biomech. 40:2367-2376, 2007. doi:10.1016 j.jbiomech.2006.12.001.

${ }^{4}$ Cheng, T., and R. Z. Gan. Mechanical properties of stapedial tendon in human middle ear. J. Biomech. Eng. 129:913-918, 2007. doi:10.1115/1.2800837.

${ }^{5}$ Ciarletta, P., S. Micera, D. Accoto, and P. Dario. A novel microstructural approach in tendon viscoelastic modeling at the fibrillar level. J. Biomech. 39:2034-2042, 2006. doi:10.1016/j.jbiomech.2005.06.025.

${ }^{6}$ Crisco, J., S. Chelikani, R. Brown, and S. Wolfe. The effect of exercise on ligamentous stiffness of the wrist. $J$. Hand Surg. 22A:44-48, 1997.

${ }^{7}$ Doehring, T. C., A. D. Freed, E. O. Carew, and I. Vesely. Fractional order viscoelasticity of the aortic valve cusp: An alternative to quasilinear viscoelasticity. J. Biomech. Eng. 127(4):700-708, 2005. doi:10.1115/1.1933900.

${ }^{8}$ Eckstrom, L., A. Kaigle, E. Hult, S. Holm, M. Rostgat, and $\mathrm{T}$. Hansson. Intervertebral disc response to cyclic loading: An animal model. Proc. Inst. Mech. Eng. 210:249258, 1996. doi:10.1243/PIME_PROC_1996_210_421_02.

${ }^{9}$ Elliot, D. M., P. S. Robinson, J. A. Gimbel, J. J. Sarver, J. A. Abboud, R. V. Iozzo, and L. J. Soslowsky. Effect of altered matrix proteins on quasilinear viscoelastic properties in transgenic mouse tail tendons. Ann. Biomed. Eng. 31:599-605, 2003. doi:10.1114/1.1567282.

${ }^{10}$ Gedalia, U., M. Solomonow, B. Zhou, R. Baratta, Y. Lu, and M. Harris. Biomechanics of increased exposure to lumbar injury caused by cyclic loading II: Recovery of reflexive muscular stability with rest. Spine 24(23):24612467, 1999. doi:10.1097/00007632-199912010-00007.

${ }^{11}$ Graf, B. K., R. Vanderby, M. J. Ulm, R. P. Rogalski, and R. J. Thielke. Effect of preconditioning on the viscoelastic response of primate patellar tendon. Arthroscopy 10(1):90 96, 1994.
${ }^{12}$ Han, S., S. J. Gemmell, K. G. Helmer, P. Grigg, J. W. Wellen, A. H. Hoffman, and C. H. Sotak. Changes in ADC caused by tensile loading of rabbit achilles tendon: Evidence for water transport. J. Mag. Reson. 144:217-227, 2000. doi:10.1006/jmre.2000.2075.

${ }^{13}$ Hingorani, R. V., P. P. Provenzano, R. S. Lakes, A. Escarcega, and R. Vanderby. Nonlinear viscoelasticity in rabbit medical collateral ligament. Ann. Biomed. Eng. 32(2):306-312, 2004. doi:10.1023/B:ABME.0000012751. 31686.70

${ }^{14}$ Hirpara, K. M., P. J. Sullivan, and M. E. O’Sullivan. The effects of freezing on the tensile properties of repaired porcine flexor tendon. J. Hand Surg. 33A:353-358, 2008. doi:10.1016/j.jhsa.2007.12.011.

${ }^{15}$ Lakes, R. S. Viscoelastic Materials. Cambridge: Cambridge University Press, 2009.

${ }^{16}$ Lakes, R. S., and R. Vanderby. Interrelation of creep and relaxation: A modeling approach for ligaments. J. Biomech. Eng. 121:612-615, 1999. doi:10.1115/1.2800861.

${ }^{17}$ Ledoux, W. R., D. F. Meaney, and H. J. Hillstrom. A quasilinear, viscoelastic, structural model of the plantar soft tissue with frequency-sensitive damping properties. J. Biomech. Eng. 126(6):831-837, 2004. doi:10.1115/1.1824133.

${ }^{18}$ Lieber, R. L., M. E. Leonard, C. G. Brown, and C. L. Trestik. Frog semitendinosis tendon load-strain and stressstrain properties during passive loading. Am. J. Phys. 261:C86-C92, 1991.

${ }^{19}$ Lin, Y., G. H. Koenderink, F. C. MacKintosh, and D. A. Weitz. Viscoelastic properties of microtubules. Macromolecules 40(21):7714-7720, 2007. doi:10.1021/ma0708621.

${ }^{20}$ Magnusson, S. P., P. Aagaard, and J. J. Nielson. Passive energy return after repeated stretches of the hamstring muscle-tendon unit. Med. Sci. Sports Exerc. 32(6):11601164, 2002. doi:10.1097/00005768-200006000-00020.

${ }^{21} \mathrm{McGill}, \mathrm{S}$., and S. Brown. Creep response of lumbar spine to prolonged full flexion. Clin. Biomech. 7:43-46, 1992. doi:10.1016/0268-0033(92)90007-Q.

${ }^{22}$ Moon, D. K., S. L. Woo, Y. Takakura, M. T. Gabriel, and S. D. Abramowitch. The effects of refreezing on the viscoelastic and tensile properties of ligaments. J. Biomech. 39:1153-1157, 2006. doi:10.1016/j.jbiomech.2005.02.012.

${ }^{23}$ Mow, V. C., A. F. Mak, W. M. Lai, L. C. Rosenberg, and L. H. Tang. Viscoelastic properties of proteoglycan subunits and aggregates in varying solution concentrations. $J$. Biomech. 17(5):325-338, 1984. doi:10.1016/0021-9290(84) 90027-7.

${ }^{24}$ Mow, V. C., W. Zhu, W. M. Lai, T. E. Hardingham, C. Hughes, and $\mathrm{H}$. Muir. The influence of link protein stabilization on the viscometric properties of proteoglycan aggregate solutions. Biochim. Biophys. Acta 992(2):201208, 1989

${ }^{25}$ Navajas, D., G. N. Maksym, and J. H. T. Bates. Dynamic viscoelastic nonlinearity of lung parenchymal tissue. J. Appl. Physiol. 79(1):348-356, 1995.

${ }^{26}$ Nekouzadeh, A., K. M. Pryse, E. L. Elson, and G. M. Genin. A simplified approach to quasilinear viscoelastic modeling. J. Biomech. 40(14):3070-3078, 2007. doi:10.1016/ j.jbiomech.2007.03.019.

${ }^{27} \mathrm{Oza}, \mathrm{A} ., \mathrm{R}$. Vanderby, and R. S. Lakes. Interrelation of creep and relaxation for nonlinearly viscoelastic materials: Application to ligament and metal. Rheol. Acta 42:557-568, 2003. doi:10.1007/s00397-003-0312-0.

${ }^{28}$ Provenzano, P., D. Heisey, K. Hayashi, R. Lakes, and R. Vanderby. Subfailure damage in ligament: A structural and cellular evaluation. J. Appl. Phys. 92:362-371, 2002. 
${ }^{29}$ Provenzano, P., R. Lakes, T. Keenan, and R. Vanderby. Nonlinear ligament viscoelasticity. Ann. Biomed. Eng. 29:908-914, 2001. doi:10.1114/1.1408926.

${ }^{30}$ Rumian, A. P., A. L. Wallace, and H. L. Birch. Tendons and ligaments are anatomically distinct but overlap in molecular and morphological features-A comparative study in an ovine model. J. Orthop. Res. 25(4):458-464, 2007. doi:10.1002/jor.20218.

${ }^{31}$ Shadwick, R. E. Elastic energy storage in tendons: Mechanical differences related to function and age. J. Appl. Physiol. 68(3):1033-1040, 1990. doi:10.1063/1.346741.

${ }^{32}$ Solomonow, M., B. H. Zhou, R. V. Baratta, Y. Lu, M. $\mathrm{Zhu}$, and $\mathrm{M}$. Harris. Biexponential recovery model of lumbar viscoelasticity and reflexive muscular activity after prolonged cyclic loading. Clin. Biomech. 15:167-175, 2000. doi:10.1016/S0268-0033(99)00062-5.

${ }^{33}$ Sverdlik, A., and Y. Lanir. Time-dependent mechanical behavior of sheep digital tendons, including the effects of preconditioning. J. Biomech. Eng. 124:78-84, 2002. doi:10.1115/1.1427699.
${ }^{34}$ van Dommelen, J. A. W., M. M. Jolandan, B. J. Ivarsson, S. A. Millington, M. Raut, J. R. Kerrigan, J. R. Crandall, and D. R. Diduch. Nonlinear viscoelastic behavior of human knee ligaments subjected to complex loading histories. Ann. Biomed. Eng. 34(6):1008-1018, 2006. doi:10.1007/s10439006-9100-1.

${ }^{35}$ Woo, S. L.-Y., M. A. Gomez, and W. H. Akeson. The time and history dependent viscoelastic properties of the canine medial collateral ligament. J. Biomech. Eng. 103:293-298, 1981.

${ }^{36}$ Yang, W., T. C. Fung, K. S. Chian, and C. K. Chong. Viscoelasticity of esophageal tissue and application of QLV model. J. Biomech. Eng. 128(6):909-916, 2006. doi:10.1115/ 1.2372473 .

${ }^{37}$ Zhang, C., and I. D. Moore. Nonlinear mechanical response of high density polyethylene. Part 1: Experimental investigation and model evaluation. Polym. Eng. Sci. 37(2):404-413, 1997. doi:10.1002/pen.11683. 